\title{
Tocotrienol regulates osteoclastogenesis in rheumatoid arthritis
}

\author{
Kyoung-Woon Kim ${ }^{1,}$, Bo-Mi Kim ${ }^{1}$, Ji-Yeon Won ${ }^{1,2,}{ }^{,}$, Hong Ki Min ${ }^{2}$, Seoung Joon Lee ${ }^{3}$, Sang-Heon Lee ${ }^{2}$, \\ and Hae-Rim $\mathrm{Kim}^{2}$
}

\begin{abstract}
${ }^{1}$ Convergent Research Consortium for Immunologic Disease, Seoul St. Mary's Hospital, College of Medicine, The Catholic University of Korea, Seoul; ${ }^{2}$ Division of Rheumatology, Department of Internal Medicine, Research Institute of Medical Science, Konkuk University School of Medicine, Seoul; ${ }^{3}$ Department of Orthopedic Surgery, Konkuk University School of Medicine, Seoul, Korea
\end{abstract}

Received: November 5, 2019 Revised : December 26, 2019 Accepted: December 30, 2019

\section{Correspondence to}

Hae-Rim Kim, M.D.

Division of Rheumatology,

Department of Internal

Medicine, Konkuk University

School of Medicine, 120-1

Neungdong-ro, Gwangjin-gu,

Seoul 05030, Korea

Tel: +82-2-2030-7542

Fax: +82-2-2030-7748

E-mail: kimhaerim@kuh.ac.kr

https://orcid.org/0000-0002-

$1911-6236$

*Current affiliation: Research

\& Development Center,

OncoInsight, Seoul, Korea
Background/Aims: The present study aimed to investigate whether tocotrienol regulates interleukin 17 (IL-17)-induced osteoclastogenesis in rheumatoid arthritis (RA).

Methods: We evaluated the effect of tocotrienol on IL-17-induced receptor activator of nuclear factor kappa B ligand (RANKL) production using RA fibroblast-like synoviocyte (FLS), together with real-time polymerase chain reaction and enzyme-linked immunosorbent assay. Osteoclast differentiation was confirmed after culturing IL-17-treated RA FLS and Th17 cells with tocotrienol and monocytes. We analyzed the suppressive effect of tocotrienol on Th17 cells percentage or Th17-cytokine levels among peripheral blood mononuclear cells using flow cytometry.

Results: We found that IL-17 stimulated FLS to produce RANKL and tocotrienol decreased this IL-17-induced RANKL production. Tocotrienol decreased the IL-17-induced activation of mammalian target of rapamycin, extracellular signal-regulated kinase, and inhibitor of kappa B-alpha. When monocytes were incubated with IL-17, RANKL, IL-17-treated FLS or Th17 cells, osteoclasts were differentiated and tocotrienol decreased this osteoclast differentiation. Tocotrienol reduced Th17 cell differentiation and the production of IL-17 and SRANKL; however, tocotrienol did not affect Treg cell differentiation.

Conclusions: Tocotrienol inhibited IL-17- activated RANKL production in RA FLS and IL-17-activated osteoclast formation. In addition, tocotrienol reduced Th17 differentiation. Therefore, tocotrienol could be a new therapeutic choice to treat bone destructive processes in RA.

Keywords: Interleukin-17; Rheumatoid arthritis; Osteoclastogenesis; Gamma-tocotrienol; RANK ligand

\section{INTRODUCTION}

Rheumatoid arthritis (RA) is a chronic systemic autoimmune disease marked by symptoms of inflammation and pain in the joints [1]. Increased production of tumor necrosis factor (TNF)- $\alpha$, elevated oxidative stress, interleukin $1 \beta$ (IL-1 $\beta$ ), and IL- 6 affect bone metabolism [2]. In RA, fibroblast-like synoviocyte (FLS) also play a key role by producing cytokines that pro- mote inflammation and proteases that contribute to cartilage and bone destruction [3]. An osteoclast is a type of bone cell that breaks down bone tissue in RA. Osteoclasts are regulated by macrophage colony-stimulating factor (M-CSF) and receptor activator of nuclear factor $\kappa-\mathrm{B}$ ligand (RANKL) and they are activated in the inflamed synovium [4].

IL-17 is one of cytokines that induce osteoclast differentiation directly. IL-17 also stimulates osteoclasto- 
genesis indirectly through induction of TNF- $\alpha$, IL-1, IL- 6 and RANKL from macrophages and FLS $[5,6]$. In our previous study, IL-17 stimulates the production of RANKL from RA FLS and differentiates peripheral blood monocytes into mature osteoclasts [7]. Although IL-17 is essential for development of inflammatory arthritis and joint destruction, the results of human clinical studies for blocking IL-17 are controversial. Ixekizumab, an anti-IL-17A monoclonal antibody, improves sign and symptoms in RA patients, decreasing disease activity score and acute phase reactant at week 12 [8]. Another IL-17 inhibitor, secukinumab, does not achieve the primary efficacy, however, it decreases RA disease activity [9]. After all the analysis, the blockage of IL-17 alone is not sufficient in control inflammation and joint destruction and the ancillary therapeutic modalities are required.

Tocotrienol, a vitamin E family, suppresses inflammatory cytokines. In several experiments, lipopolysaccharides-induced production of inflammatory cytokines and nitric oxide are decreased by tocotrienol [10-12]. In addition, tocotrienol regulates the disease severity of arthritis in the arthritis animal models [13,14]. Moreover, tocotrienol suppresses osteoclast differentiation in mouse bone marrow-derived macrophages by suppression of nuclear factor of activated T cells 1 (NFAT1) and Fos proto-oncogene, AP-1 transcription factor subunit (c-Fos) $[15,16]$ and in tartrate-resistant acid phosphatase (T'RAP)+ cells from human CD14 $4^{+}$monocytes [17]. Until now, there is no study showing the effect of tocotrienol in IL-17-induced osteoclastogenesis. Moreover, the effect of tocotrienol has not been studied on human disease cells such as RA FLS.

Thus, the present study aimed to: (1) detect the regulatory effect of tocotrienol on IL-17-induced RANKL expression and osteoclast differentiation; (2) evaluate whether tocotrienol suppresses osteoclast differentiation when osteoclast precursors are incubated with IL17-treated RA FLSs and Th17 cells; and (3) determine the role of tocotrienol in Th17 cell differentiation.

\section{METHODS}

\section{Ethics statement and patients}

Informed consent was obtained from all patients, and the experimental protocol was approved by the Institutional Review Board for Human Research, Konkuk University Hospital (KUH1010186), as described previously [18].

\section{Isolation of RA FLS}

RA FLS were isolated by enzymatic digestion of synovial tissues obtained from patients with RA undergoing total knee replacement surgery, who fulfilled the American College of Rheumatology (ACR) classification of rheumatoid arthritis of the knee and had advanced rheumatoid arthritis with Kellgren-Lawrens grade 4.

\section{Reagents}

Recombinant human RANKL (rhRANKL), rhM-CSF, and IL-17 were purchased from R\&D Systems (Minneapolis, MN, USA). Tocotrienol was purchased from Sigma Chemical Co. (St. Louis, MO, USA).

\section{Isolation of peripheral blood mononuclear cells}

PBMC were obtained from heparinized blood using Ficoll-Hypaque (GE Healthcare, Philadelphia, PA, USA) density-gradient centrifugation.

\section{Induction of Th17 differentiation}

To induce Th17 differentiation, peripheral blood mononuclear cells (PBMCs) were cultured for 48 hours with anti-CD28 and anti-CD3 (BD, Franklin Lakes, NJ, USA), IL-23, IL-6, IL-1 $\beta$, IL-4-blocking antibodies, and interferon-gamma (IFN- $\gamma$ )-blocking antibodies. To investigate the suppressive effects of tocotrienol, PBMCs were cultured for 3 hours with tocotrienol, and then incubated as in Th17 differentiation.

\section{Expression of RANKL mRNA by real-time polymerase chain reaction}

RA FLS were stimulated with IL-17. For RANKL signal pathway analysis, the FLSs were cultured with or without tocotrienol for 3 hours before the addition of IL-17. After stimulation for 72 hours, mRNA was extracted using RNAzol B (Biotex Laboratories, Houston, TX, USA) according to the manufacturer's instructions, and then subjected to real-time polymerase chain reaction.

\section{Enzyme-linked immunosorbent assay}

Sandwich enzyme-linked immunosorbent assays (ELISAs) were used to quantify sRANKL, IL-1 $\beta$, TNF- $\alpha$, IL-6, 

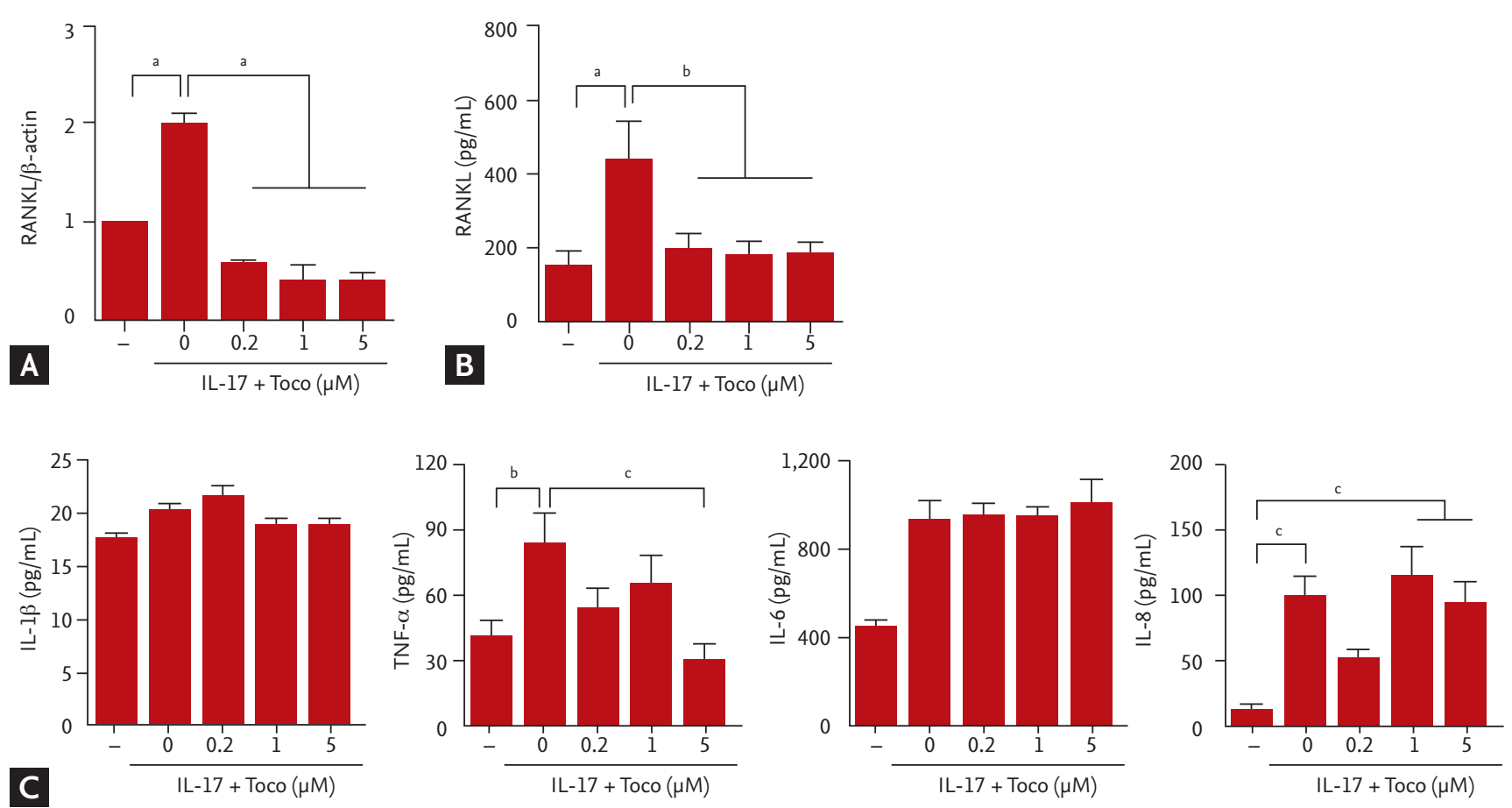

Figure 1. Effect of tocotrienol on receptor activator of nuclear factor $\kappa-B$ ligand (RANKL) expression and the production in rheumatoid arthritis (RA) fibroblast-like synoviocyte (FLS). (A) RA FLS were pretreated with various doses of tocotrienol, and then cultured with $20 \mathrm{ng} / \mathrm{mL}$ interleukin 17 (IL-17) for 72 hours. The RANKL mRNA level was quantified using real-time polymerase chain reaction. (B) RA FLS were treated with IL-17 in the presence of tocotrienol for 72 hours and the production of soluble RANKL in the cultured media was assessed using enzyme-linked immunosorbent assay (ELISA). (C) RA FLS were treated with IL-17 in the presence of tocotrienol for 72 hours and the production of IL-1 $\beta$, tumor necrosis factor $\alpha$ (TNF- $\alpha$ ), IL-6, and IL-8 were assessed using ELISA. The data represent the means \pm SEM of three independent experiments. TNF, tumor necrosis factor; Toco, tocotrienol. ${ }^{\mathrm{a}} \mathrm{p}<0.001,{ }^{\mathrm{b}} \mathrm{p}<0.05$, and ${ }^{\mathrm{c}} \mathrm{p}<0.01$.

IL-8, and IL-17 levels in the culture supernatants from RA FLS or PBMC.

\section{Western blotting analysis}

Primary antibodies recognizing to the phosphorylated forms of mammalian target of rapamycin kinase (mTOR), adenosine monophosphate (AMP)-activated protein kinase (AMPK), JUN N-terminal kinase (JNK), extracellular signalregulated kinase (ERK), or nuclear factor kappa B inhibitor alpha (IкB $\alpha$; Cell Signaling Technology Inc., Danvers, MA, USA) were diluted 1:1,000 in 0.1\% Tween 20/1x Tris-buffered saline (TTBS), and incubated overnight at $4^{\circ} \mathrm{C}$. The membranes were washed with TTBS, horseradish peroxidase-conjugated secondary antibody was added, and the membranes were incubated for 1 hour at room temperature. After washing with T'TBS, the hybridized bands were detected using an ECL detection kit (Amersham Pharmacia, Piscataway, NJ, USA).

\section{Flow cytometry analysis}

Cells were stained with combinations of the following mAbs: anti-CD4-PE/cyanine7 (Cy7) and anti-CD25-allophycocyanin (APC; BD). Cells were washed, fixed, permeabilized, and stained to detect intracellular cytokines with $\mathrm{mAbs}$ recognizing IL-17 and forkhead box $\mathrm{P}_{3}$ (FOXP 3 ; eBioscience, San Diego, CA, USA). Cells were analyzed on a FACS Calibur flow cytometry system (BD).

\section{Osteoclast formation}

Peripheral blood CD14+ monocytes were cultured with IL-17-treated FLS or Th17-polarizing T cells in media. Monocytes were cocultured in $\alpha$-minimal essential medium and $10 \% \mathrm{FBS}$ in the presence of $25 \mathrm{ng} / \mathrm{mL}$ of rhMCSF. The addition of rhRANKL was used as a positive control, which was prepared as described previously [19]. A TRAP Staining Kit was used to stain TRAP in osteoclasts. 


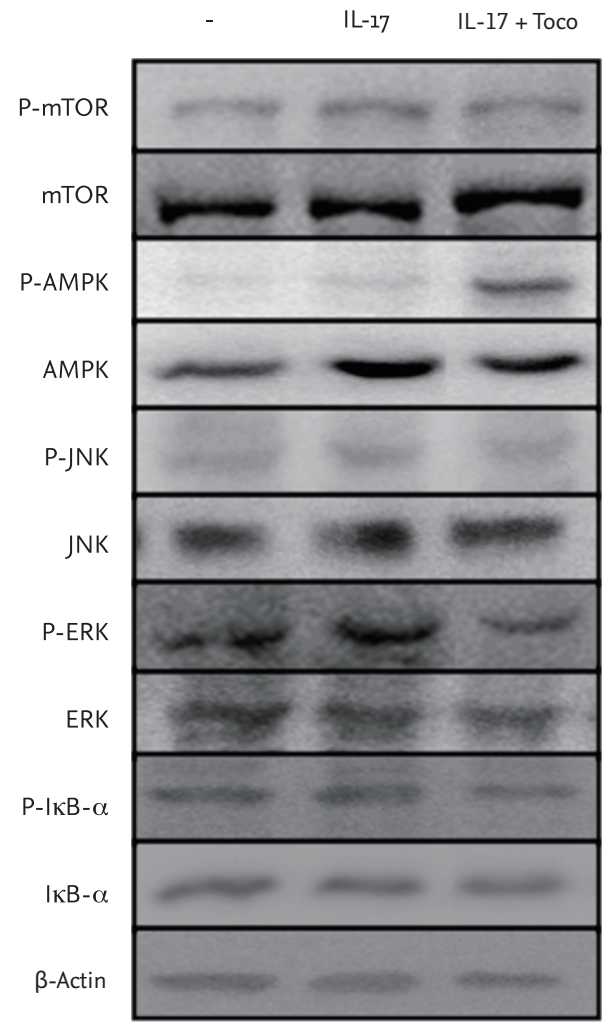

A
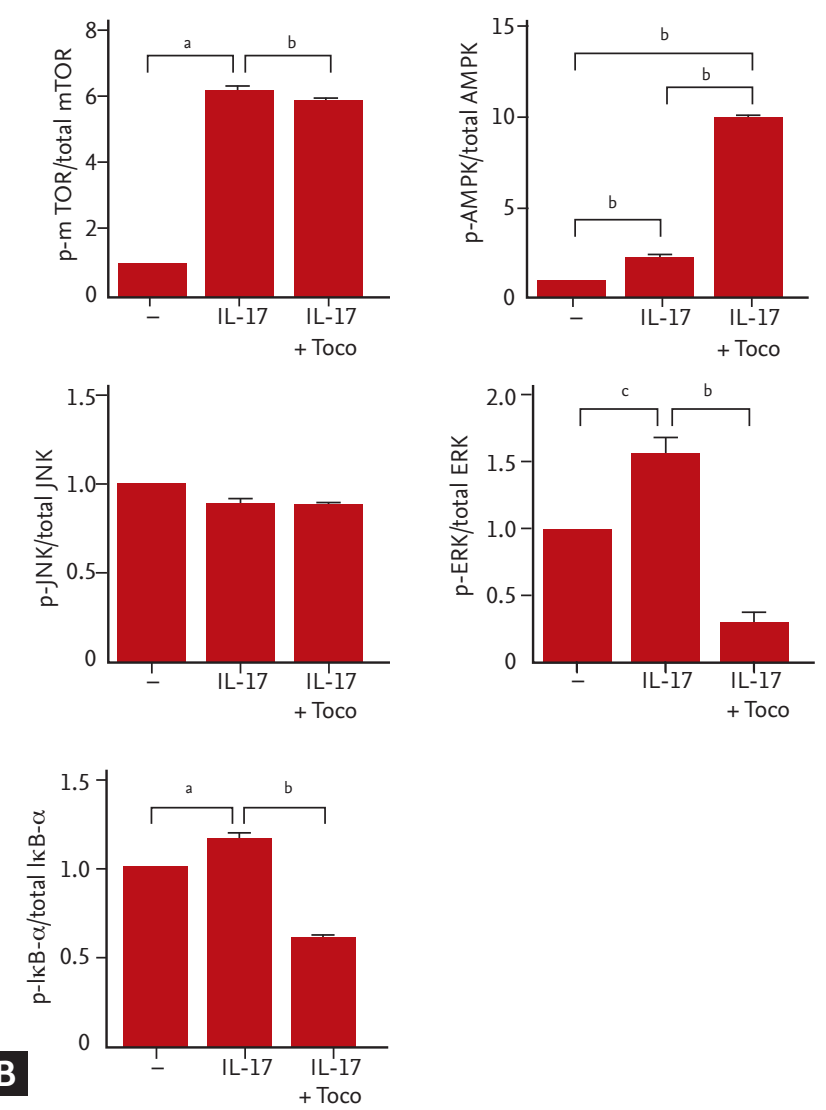

Figure 2. Effects of tocotrienol on the expression of signal pathway molecules in rheumatoid arthritis (RA) fibroblast-like synoviocyte (FLS). (A) RA FLS were pretreated with tocotrienol for 3 hours, and then cultured with 20 ng/mL interleukin 17 (IL-17) for 1 hour. The phosphorylated forms of target proteins and beta-actin were detected using Western blotting. The figures are representative of three independent experiments. (B) Data were normalized to the beta-actin level and reported in relative expression units. The data represent the mean \pm SEM of three independent experiments. Toco, tocotrienol; mTOR, mammalian target of rapamycin kinase; AMPK, AMP-activated protein kinase; JNK, JUN N-terminal kinase; ERK, extracellular signalregulated kinase; Iא $\mathrm{B} \alpha$, nuclear factor kappa B inhibitor alpha. ${ }^{\mathrm{a}} p<0.01,{ }^{\mathrm{b}} p<0.001$, and ${ }^{\mathrm{c}} p<0.05$.

\section{Statistical analysis}

The data are expressed as mean \pm standard error mean (SEM). Statistical difference was assessed using Mann-Whitney $U$ test for analyzing two groups or oneway analysis of variance with Bonferroni's multiple comparison post hoc test for analyzing more than three groups. A p value $<0.05$ was considered statistically significant.

\section{RESULTS}

\section{The suppressive effect of tocotrienol on the} IL-17-activated RANKL gene and protein in RA FLS

To detect the suppressive effect of tocotrienol on the IL-17-activated RANKL mRNA level, RA FLS were cul- tured with tocotrienol for 3 hours. The RA FLS were then cultured with $20 \mathrm{ng} / \mathrm{mL}$ IL-17 for 72 hours. IL-17 increased RANKL gene expression to its maximum extent at a dose of $20 \mathrm{ng} / \mathrm{mL}$; therefore, we used to IL-17 (20 $\mathrm{ng} / \mathrm{mL}$ ) as an optimal stimulatory dose (data not shown). Tocotrienol suppressed the IL-17-activated expression of RANKL (Fig. 1A). Tocotrienol also reduced the production of the RANKL protein with a similar pattern to its mRNA expression (Fig. 1B). Tocotrienol significantly decreased the production of TNF- $\alpha$, but did not affect the IL-17-activated production of IL-6 and IL-8 (Fig. 1C).

\section{Signal pathways involved in the protective effect of tocotrienol in RA FLS}

We investigated the intracellular signal pathways of tocotrienol in the IL-17-induced stimulation of RA FLS. 

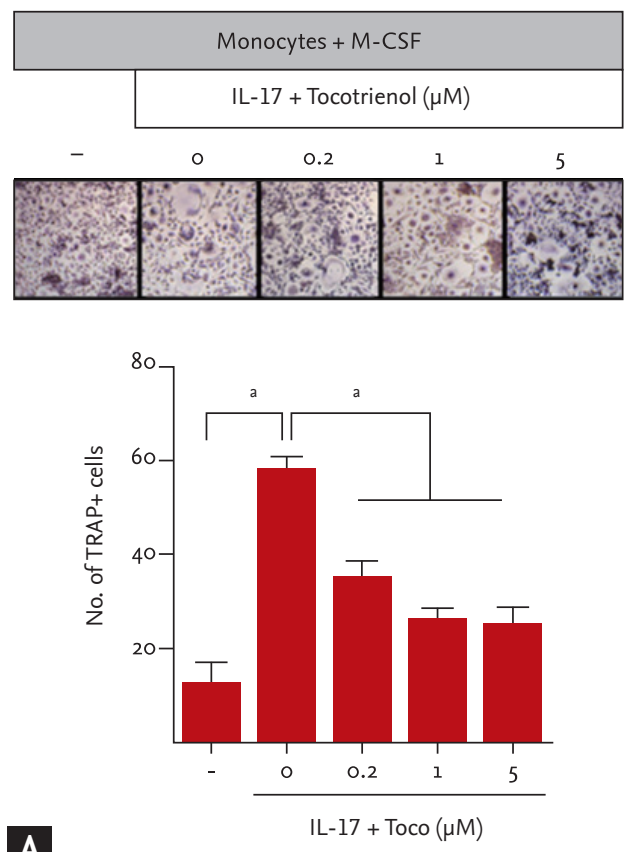

A
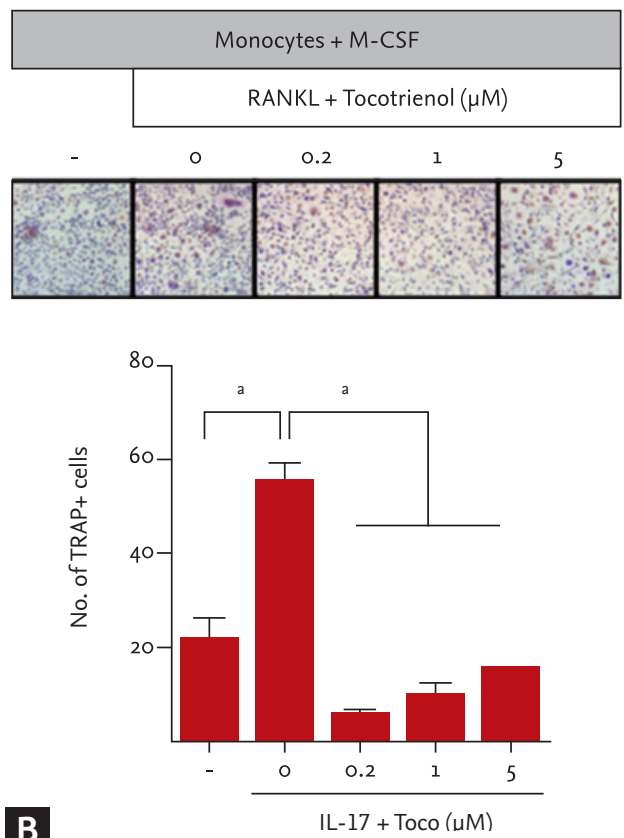

B
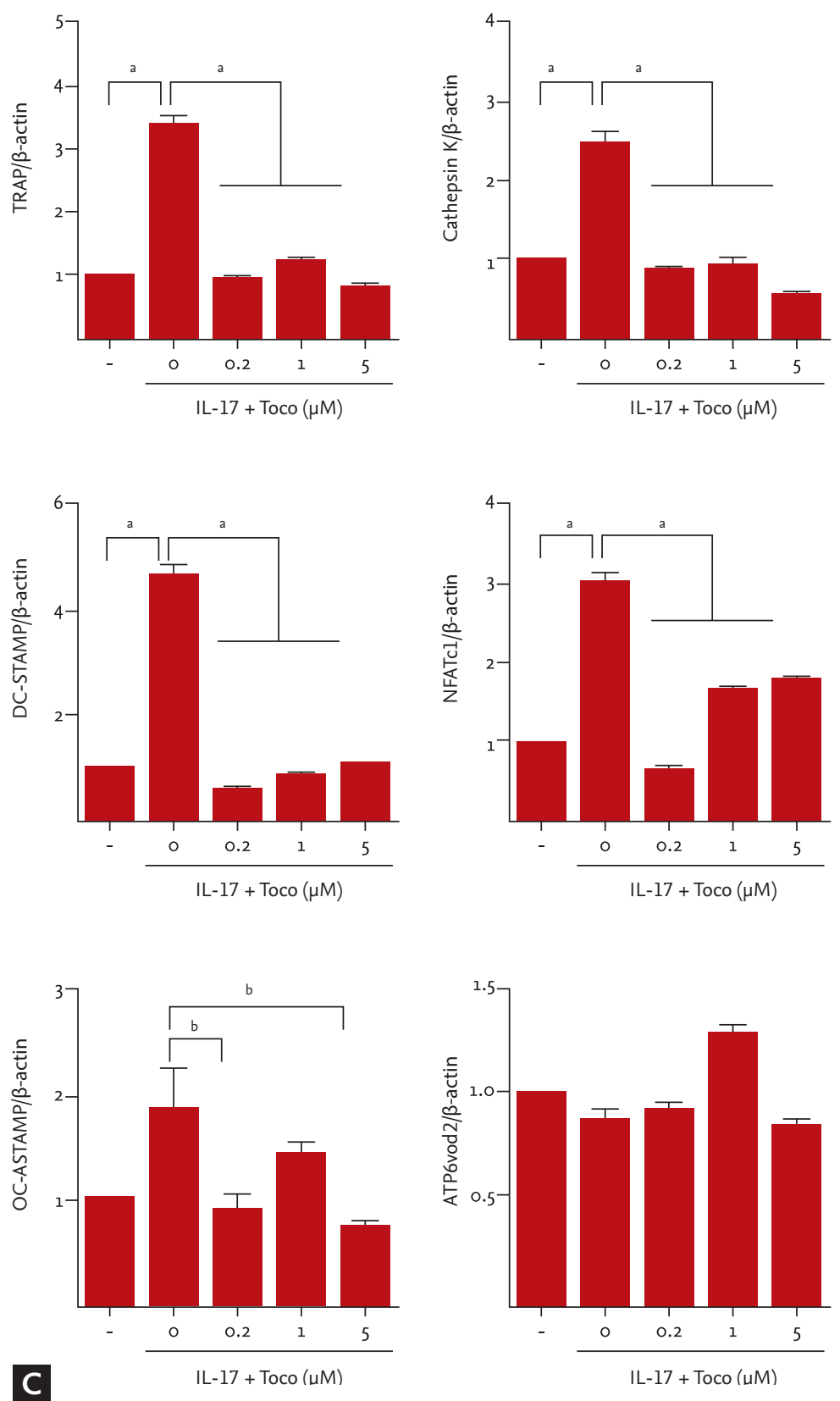

Figure 3. The effect of tocotrienol on osteoclast differentiation from peripheral blood monocytes. $\mathrm{CD}_{14}{ }^{+}$monocytes were isolated from the peripheral blood of healthy donors and then pretreated with tocotrienol for 3 hours, before being cultured with $25 \mathrm{ng} / \mathrm{mL}$ of macrophage colony-stimulating factor (M-CSF), (A) $20 \mathrm{ng} / \mathrm{mL}$ of interleukin 17 (IL-17), or (B) $30 \mathrm{ng} / \mathrm{mL}$ of receptor activator of nuclear factor $\kappa-B$ ligand (RANKL). Osteoclasts were identified using tartrate-resistant acid phosphatase (TRAP) staining. The figures are representative of three independent experiments (original magnification $\times 100)$. (C) The expression levels of genes encoding TRAP, cathepsin K, dendritic cell-specific transmembrane protein (DC-STAMP), nuclear factor of activated T cells, cytoplasmic i (NF-ATc1), osteoclast stimulatory transmembrane protein (OC-STAMP), and V-type proton ATPase subunit D2 (ATP6vod2) from differentiated osteoclasts were measured using real-time polymerase chain reaction. Data were normalized to the beta-actin mRNA level and reported in relative expression units. The data represent the mean \pm SEM of three independent experiments. Toco, tocotrienol. ${ }^{\mathrm{a}} \mathrm{p}<0.001,{ }^{\mathrm{b}} \mathrm{p}<0.05$. 

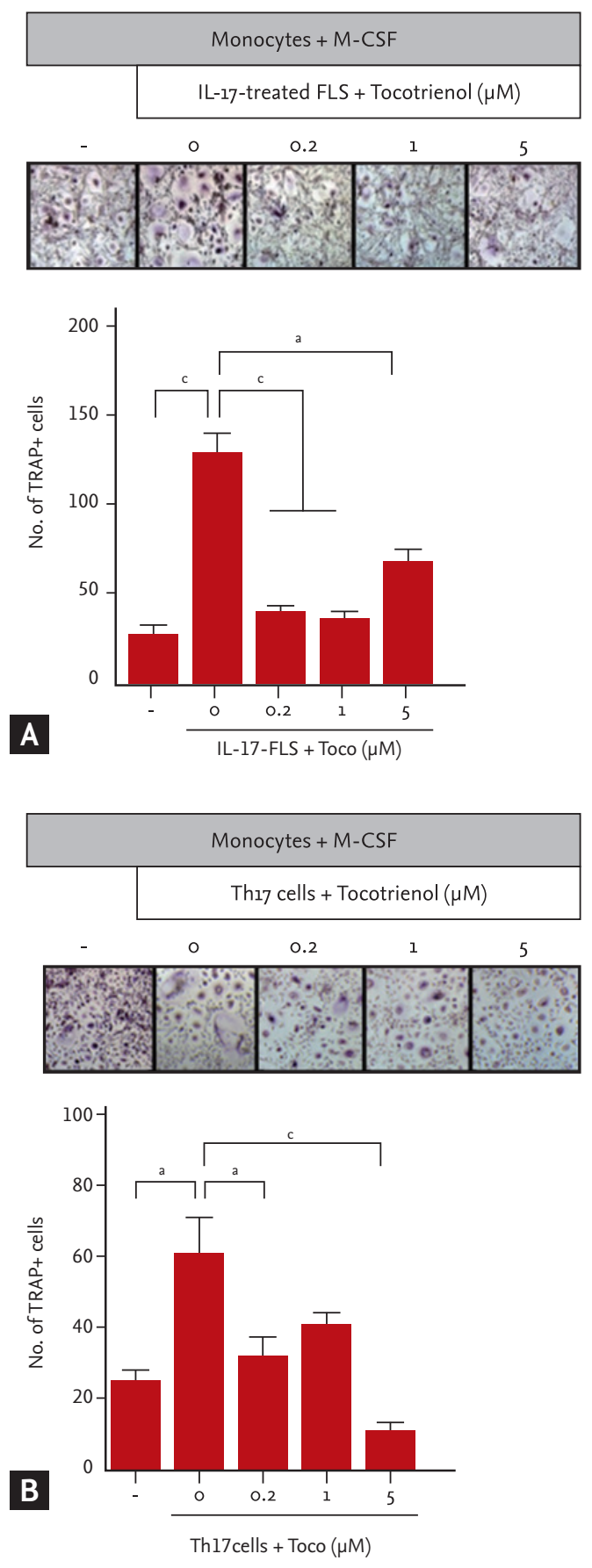
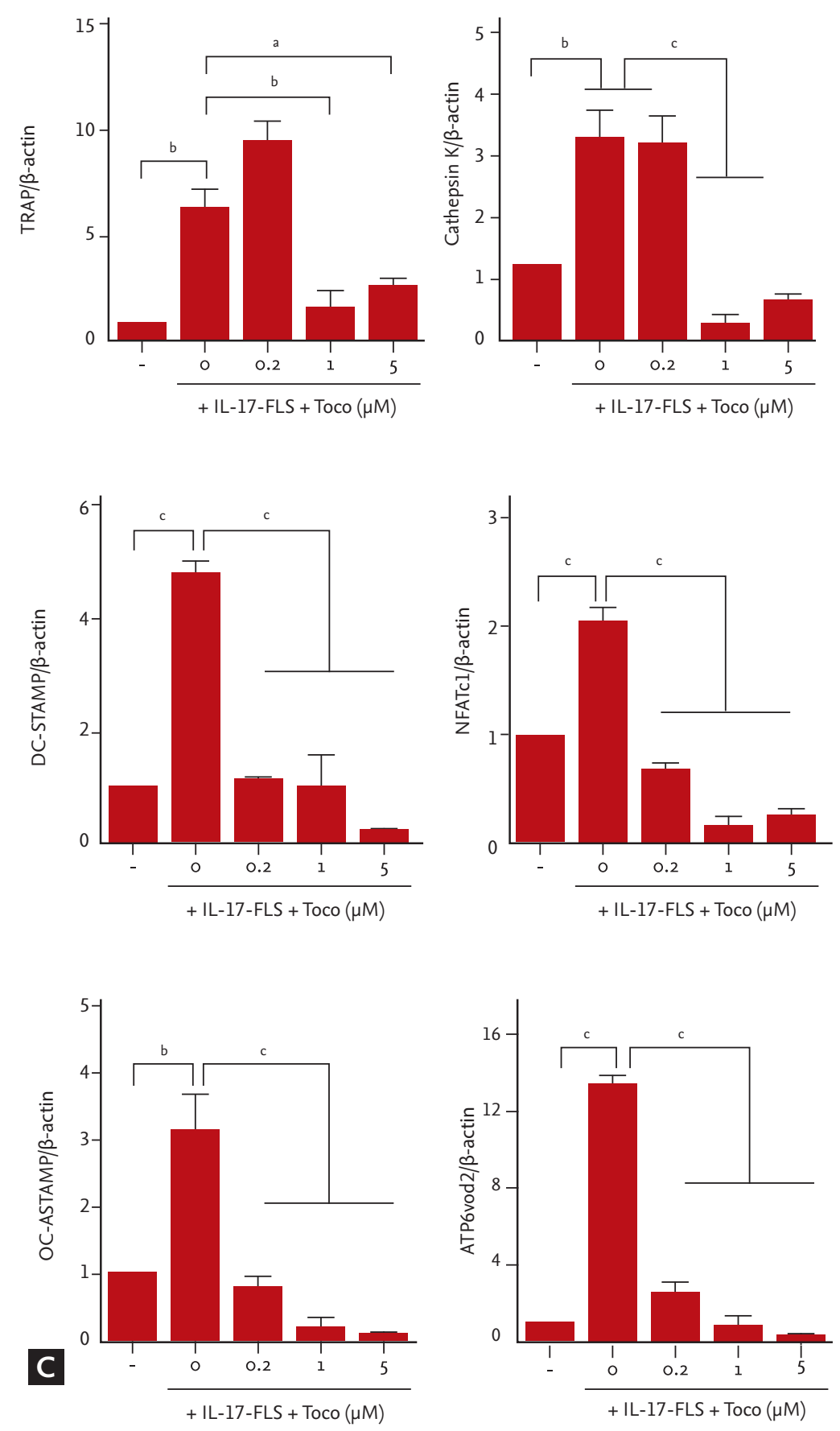

Figure 4. The effect of tocotrienol on osteoclastogenesis by interleukin 17 (IL-17)-pretreated rheumatoid arthritis (RA) fibroblast-like synoviocyte (FLS). (A) RA FLS were pretreated with tocotrienol for 3 hours, cultured with $20 \mathrm{ng} / \mathrm{mL}$ of IL-17 for 72 hours, and then cocultured with $\mathrm{CD}_{1}{ }^{+}$monocytes from peripheral blood in the presence of macrophage colony-stimulating factor (M-CSF). After 21 days of culture, tartrate-resistant acid phosphatase (TRAP)-positive multinucleated cells were counted. (B) Th17 cells were pretreated with tocotrienol for 3 hours, and then cocultured with $\mathrm{CD}_{1} 4^{+}$monocytes from peripheral blood in the presence of M-CSF. The figures are representative of three independent experiments (original magnification $\times 100$ ). (C) The expression levels of genes encoding TRAP, cathepsin K, dendritic cell-specific transmembrane protein (DC-STAMP), nuclear factor of activated T cells, cytoplasmic I (NF-ATc1), osteoclast stimulatory transmembrane protein (OC-STAMP), and ATP6vod2 from differentiated osteoclasts were measured using real-time polymerase chain reaction. Data were normalized to the beta-actin mRNA level and reported in relative expression units. The data represent the mean \pm SEM of three independent experiments. ${ }^{a} p<0.05,{ }^{b} p<0.01$, and ${ }^{c} p<0.001$. 


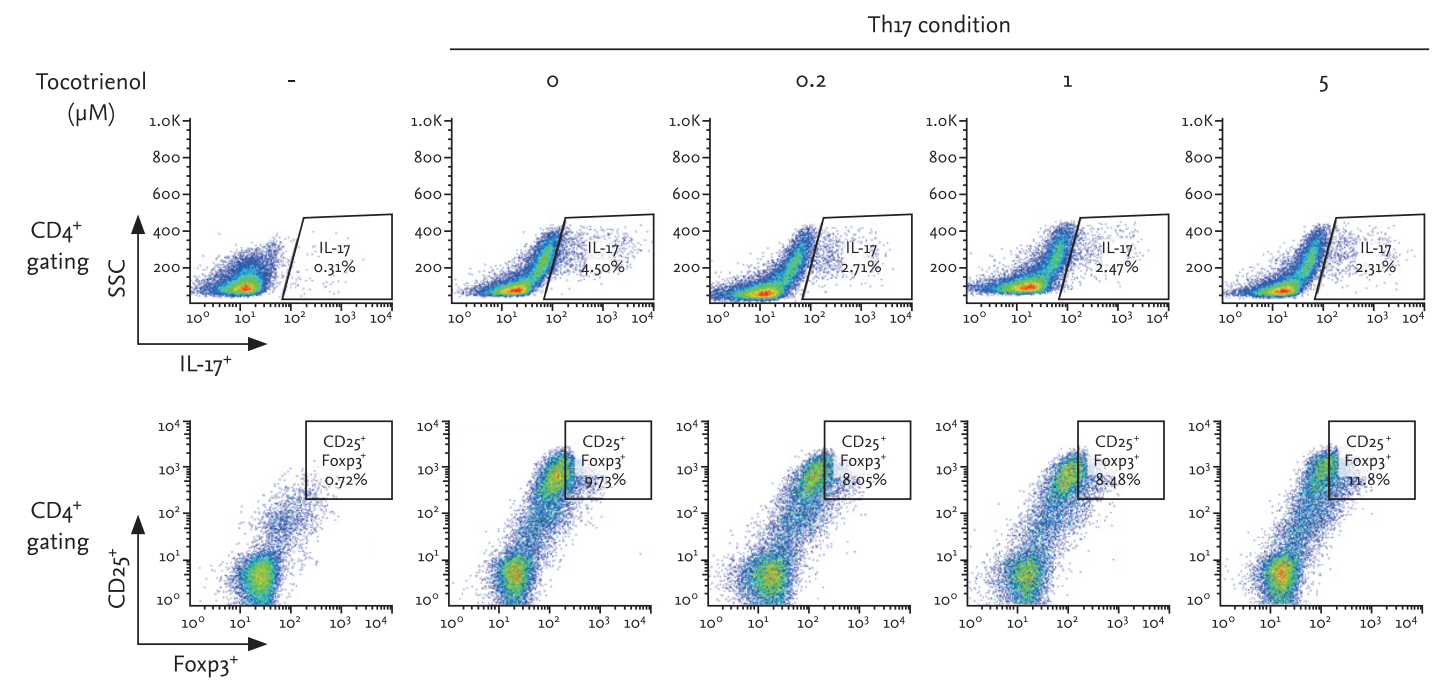

A
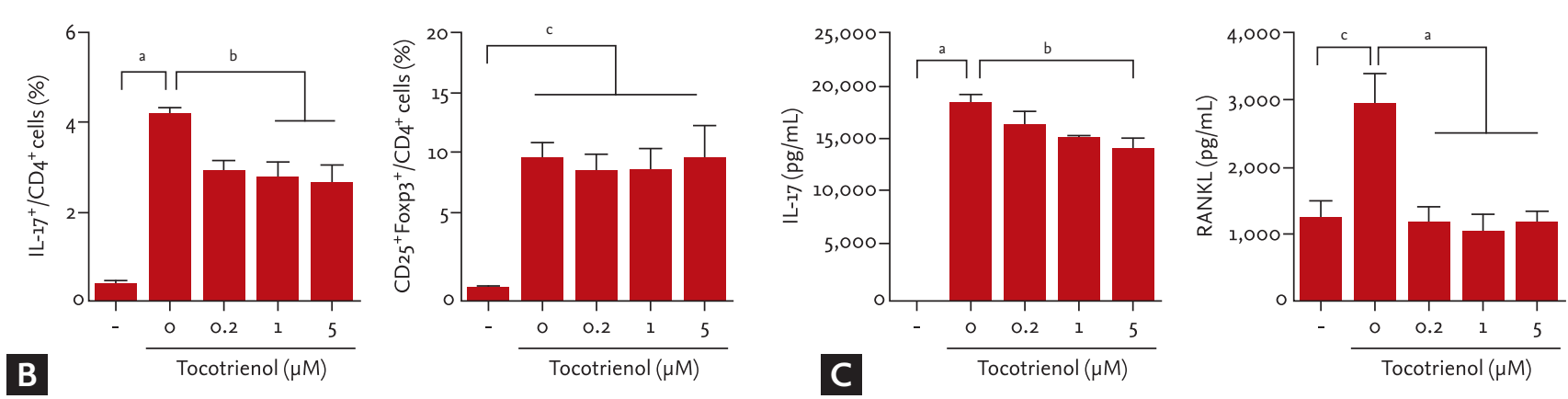

Figure 5. Effect of tocotrienol on $\mathrm{CD}_{4}{ }^{+} \mathrm{T}$ cells isolated from the peripheral blood mononuclear cell (PBMC) of healthy donors and cultured under Th17-polarizing conditions. Human PBMC were isolated from six healthy subjects and pre-incubated with tocotrienol (o, O.2, 1, and $5 \mu \mathrm{M})$ for 3 hours, and then cultured under Th17-polarizing conditions for 48 hours. (A, B) CD $4{ }^{+}$ $\mathrm{T}$ cells were gated for further analysis. The percentages of interleukin $17^{+}\left(\mathrm{IL}-17^{+}\right) / \mathrm{CD}_{4}{ }^{+} \mathrm{T}$ cells and $\mathrm{CD}_{2} 5^{+} \mathrm{Foxp}^{+} / \mathrm{CD}_{4}{ }^{+} \mathrm{T}$ cells were measured using flow cytometry. The figures are representative of three independent experiments. (C) The production of IL-17 by Th17-polarized $\mathrm{CD}_{4}^{+} \mathrm{T}$ cells and its secretion into the culture supernatant as measured using enzyme-linked immunosorbent assay (ELISA). Bars represent the mean \pm SEM. SSC, side scatter; RANKL, receptor activator of nuclear factor $\kappa-B$ ligand. ${ }^{\mathrm{a}} \mathrm{p}<0.001,{ }^{\mathrm{b}} \mathrm{p}<0.05$, and ${ }^{\mathrm{c}} \mathrm{p}<0.01$.

IL-17 enhanced the protein levels of AMPK, the phosphorylation of mTOR), ERK, and IкB- $\alpha$. Tocotrienol significantly reduced the IL-17-activated phosphorylation of mTOR, ERK, and IкB- $\alpha$ ( $p<0.05$ for each). By contrast, IL-17 did not phosphorylate JNK and tocotrienol significantly increased the IL-17-activated phosphorylation of AMPK (Fig. 2).

\section{The suppressive effect of tocotrienol in IL-17- and RANKL-activated osteoclast formation}

The isolated $\mathrm{CD}_{1} 4^{+}$monocytes from peripheral blood of three healthy volunteers were incubated with M-CSF and IL-17, which resulted in TRAP ${ }^{+}$multinucleated os- teoclast differentiation. However, pre-incubation with tocotrienol significantly reduced IL-17-activated osteoclastogenesis (Fig. 3A). To detect the suppressive effects of tocotrienol on RANKL-activated osteoclast formation, $\mathrm{CD}_{1} 4^{+}$monocytes were incubated with M-CSF and RANKL. After 21 days of culture, the cells had differentiated into osteoclasts. However, pre-incubation with tocotrienol significantly reduced RANKL-induced osteoclastogenesis (Fig. 3B). The gene expression of osteoclast markers, such as T'RAP, cathepsin K, dendritic cell-specific transmembrane protein (DC-STAMP), nuclear factor of activated T cells, cytoplasmic 1 (NF-ATc1), and osteoclast stimulatory transmembrane protein 
(OC-STAMP) increased after IL-17 stimulation; however, their expression was reduced by tocotrienol treatment (Fig. $3 \mathrm{C}$ ).

\section{The inhibitory effect of tocotrienol in osteoclast formation with coculture of monocytes in addition to RA FLS}

RA FLS were incubated with IL-17 and tocotrienol, and then they were cocultured with peripheral blood $\mathrm{CD}_{1}{ }^{+}$ monocytes and M-CSF. When the monocytes were cocultured with IL-17-activated RA FLS without RANKL, TRAP $^{+}$multinucleated osteoclasts differentiated and osteoclastogenesis was more pronounced compared with monocytes that were cocultured with untreated RA FLS (Fig. 4A). Tocotrienol suppressed the differentiation of osteoclasts induced by Th17 cells (Fig. 4B). The gene expression of osteoclast markers increased during coculture with IL-17-pretreated RA FLS, while tocotrienol treatment decreased their mRNA expression (Fig. 4C).

\section{The suppressive effects of tocotrienol on Th17 cell differentiation}

PBMCs were isolated from normal individuals and incubated under Th17-polarizing conditions in the presence of tocotrienol, and the percent of IL-17 positive and Foxp3 positive cells were counted using flow cytometry. In Th17-polarizing conditions, both Th17 cells and Treg cells were differentiated, and tocotrienol remarkably decreased the proportion of $\mathrm{IL}-17^{+} / \mathrm{CD}_{4}{ }^{+} \mathrm{T}$ cells. However, tocotrienol did not affect the differentiation of the cells into $\mathrm{CD}_{25}{ }^{+} \mathrm{Foxp}_{3}{ }^{+} / \mathrm{CD}_{4}{ }^{+}$regulatory $\mathrm{T}$ cells (Fig. $5 \mathrm{~A}$ and $\left.{ }_{5} \mathrm{~B}\right)$. Under Th17-polarizing condition, the production of IL-17 and RANKL increased; however, tocotrienol decreased the production of IL-17 and sRANKL in the culture supernatant (Fig. ${ }_{5} \mathrm{C}$ ).

\section{DISCUSSION}

In this study, we confirmed that tocotrienol decreased IL-17-activated RANKL gene expression and protein production in RA FLS, and inhibited IL-17-induced osteoclast differentiation. IL-17 is an important cytokine-associated with osteoclast formation. Previously, we found that other Th17 cytokines induce RANKL expression in RA FLS $[7,20]$. Under Th17-polarizing con- ditions, tocotrienol reduced Th17 cell differentiation and the production of IL-17 and sRANKL, but did not affect Treg cell differentiation. RANKL and M-CSF are essential cytokines for osteoclast differentiation from precursor cells [21]. This result suggested that tocotrienol indirectly interrupts the initial process of the Th17 cytokine-associated bony destructive pathways through inhibition of RANKL production.

In terms of signal transduction pathways, we examined the molecular signaling pathways associated with the regulatory effects of tocotrienol on RA FLS. We focused on the mTOR/AMPK/JNK/ERK/IKB- $\alpha$ pathway, which has an essential role in the activation of RA FLS [18]. In signaling pathways, the suppressive effects of tocotrienol are mediated through the mTOR/JNK/nuclear factor $\kappa \mathrm{B}(\mathrm{NF}-\kappa \mathrm{B})$ pathway [22,23]. IL-17 activates mTOR, and tocotrienol decreased IL-17-activated mTOR phosphorylation. In addition, tocotrienol increased AMPK phosphorylation. In previous studies, $\gamma$-tocotrienol demonstrated an inhibitory effect on the NF- $\mathrm{\kappa B}$ activation pathway [24-27]. Moreover, Wu et al. [28] identified that a tocotrienol-rich fraction was capable of inhibiting proinflammatory cytokine production in human monocytes.

We examined the direct inhibitory effect of tocotrienol on the formation of osteoclasts from their precursors. Tocotrienol directly inhibited RANKL and IL-17-activated osteoclast differentiation in a dose-dependent manner. This result suggested that tocotrienol could directly ameliorate cytokine-induced osteoclastogenesis, indicating its possible therapeutic use.

Next, we determined whether tocotrienol modulates Th17 cells under direct stimulation of osteoclast formation. When monocytes were cultured with Th17 cells, osteoclast differentiation increased compared with that in cells cocultured with $\mathrm{CD}_{4}{ }^{+} \mathrm{T}$ cells. Without RANKL, Th17 cells directly induced osteoclast differentiation and tocotrienol inhibited this Thry cellinduced osteoclastogenesis. Tocotrienol controls Th17 cell-induced osteoclastogenesis in different ways. First, tocotrienol inhibited IL-17-induced RANKL expression in RAFLSs. Second, tocotrienol reduced IL-17-induced osteoclast differentiation. Third, tocotrienol inhibited Thr7 cell-induced osteoclast differentiation. The ultimate goal of medical care in patients with RA is the prevention of joint destruction. Bone erosion caused by activat- 
ed osteoclasts induces joint destruction and patient disability. The results of the present study suggested that tocotrienol could prevent the bony destructive process in RA.

There are some limitations in this study. First, we observed the effect of tocotrienol only in vitro. If preclinical animal study with tocotrienol is performed, more information of therapeutic effect of tocotrienol is produced. Second, we evaluated the effect of tocotrienol using a single target cell, FLS, however, macrophages/monocytes are major cells producing TNF- $\alpha$, IL-1 and IL-6. The effect of tocotrienol to the macrophages/monocytes needs to be assessed. Third, tocotrienol reduced IL-17induced production of TNF- $\alpha$; however, we did not analyze the inhibitory mechanism and we did not focus on TNF- $\alpha$.

In conclusion, tocotrienol significantly suppressed IL-17-activated RANKL expression and osteoclast differentiation, and attenuated Thr 7 differentiation. The results suggested that tocotrienol could represent a new therapeutic choice to prevent osteoclast-associated bony destructive diseases, such as RA.

\section{KEY MESSAGE}

1. Tocotrienol decreased interleukin (IL)-17-induced receptor activator of nuclear factor $\kappa-B$ ligand (RANKL) production in rheumatoid arthritis (RA) fibroblast-like synoviocyte (FLS).

2. Tocotrienol decreased the IL-17-induced activation of mammalian target of rapamycin, extracellular signal-regulated kinase, and inhibitor of kappa B-alpha.

3. When monocytes were incubated with IL-17, RANKL, IL-17-treated FLS, or Th17 cells, osteoclasts were produced, and tocotrienol decreased this osteoclast differentiation.

4. Tocotrienol reduced Th17 cell differentiation and the production of IL-17 and SRANKL; however, tocotrienol did not affect Treg cell differentiation.

5. Tocotrienol could be a new therapeutic choice to treat bone destructive processes in RA.

\section{Conflict of interest}

No potential conflict of interest relevant to this article was reported.

\section{Acknowledgments}

This research was supported by a grant of the Basic Science Research Program through the National Research Foundation of Korea (NRF) funded by the Ministry of Education, Science and Technology, Republic of Korea (NRF-2018R1D1A1A02050982) and the Basic Science Research Program through the National Research Foundation of Korea funded by the Ministry of Science, ICT \& Future Planning (NRF-2018R1A2B2006820).

\section{REFERENCES}

1. Harris ED Jr. Rheumatoid arthritis. Pathophysiology and implications for therapy. N Engl J Med 1990;322:12771289.

2. Rosen CJ, Bouxsein ML. Mechanisms of disease: is osteoporosis the obesity of bone? Nat Clin Pract Rheumatol 2006;2:35-43.

3. Bartok B, Firestein GS. Fibroblast-like synoviocytes: key effector cells in rheumatoid arthritis. Immunol Rev 2010;233:233-255.

4. Gravallese EM. Bone destruction in arthritis. Ann Rheum Dis 2002;61 Suppl 2:ii84-ii86.

5. Lubberts E, van den Bersselaar L, Oppers-Walgreen B, et al. IL-17 promotes bone erosion in murine collagen-induced arthritis through loss of the receptor activator of NF-kappa B ligand/osteoprotegerin balance. J Immunol 2003;170:2655-2662.

6. Jovanovic DV, Di Battista JA, Martel-Pelletier J, et al. IL17 stimulates the production and expression of proinflammatory cytokines, IL-beta and TNF-alpha, by human macrophages. J Immunol 1998;160:3513-3521.

7. Kim KW, Kim HR, Kim BM, Cho ML, Lee SH. Th17 cytokines regulate osteoclastogenesis in rheumatoid arthritis. Am J Pathol 2015;185:3011-3024.

8. Genovese MC, Greenwald M, Cho CS, et al. A phase II randomized study of subcutaneous ixekizumab, an anti-interleukin-17 monoclonal antibody, in rheumatoid arthritis patients who were naive to biologic agents or had an inadequate response to tumor necrosis factor inhibitors. Arthritis Rheumatol 2014;66:1693-1704. 
9. Genovese MC, Durez P, Richards HB, et al. Efficacy and safety of secukinumab in patients with rheumatoid arthritis: a phase II, dose-finding, double-blind, randomised, placebo controlled study. Ann Rheum Dis 2013;72:863-869.

10. Qureshi AA, Tan X, Reis JC, et al. Inhibition of nitric oxide in LPS-stimulated macrophages of young and senescent mice by $\delta$-tocotrienol and quercetin. Lipids Health Dis 2011;10:239.

11. Yam ML, Abdul Hafid SR, Cheng HM, Nesaretnam K. Tocotrienols suppress proinflammatory markers and cyclooxygenase-2 expression in RAW264.7 macrophages. Lipids 2009;44:787-797.

12. Qureshi AA, Reis JC, Papasian CJ, Morrison DC, Qureshi N. Tocotrienols inhibit lipopolysaccharide-induced pro-inflammatory cytokines in macrophages of female mice. Lipids Health Dis 2010;9:143.

13. Radhakrishnan A, Tudawe D, Chakravarthi S, Chiew GS, Haleagrahara N. Effect of $\gamma$-tocotrienol in counteracting oxidative stress and joint damage in collagen-induced arthritis in rats. Exp Ther Med 2014;7:1408-1414.

14. Haleagrahara N, Swaminathan M, Chakravarthi S, Radhakrishnan A. Therapeutic efficacy of vitamin E $\delta$-tocotrienol in collagen-induced rat model of arthritis. Biomed Res Int 2014;2014:539540.

15. Ha H, Lee JH, Kim HN, Lee ZH. $\alpha$-Tocotrienol inhibits osteoclastic bone resorption by suppressing RANKL expression and signaling and bone resorbing activity. Biochem Biophys Res Commun 2011;406:546-551.

16. Lee JH, Kim HN, Yang D, et al. Trolox prevents osteoclastogenesis by suppressing RANKL expression and signaling. J Biol Chem 2009;284:13725-13734.

17. Brooks R, Kalia P, Ireland DC, Beeton C, Rushton N. Direct inhibition of osteoclast formation and activity by the vitamin E isomer gamma-tocotrienol. Int J Vitam Nutr Res 2011;81:358-367.

18. Kim HR, Kim KW, Kim BM, Lee KA, Lee SH. N-acetyl-1-cysteine controls osteoclastogenesis through regulating Th17 differentiation and RANKL production in rheumatoid arthritis. Korean J Intern Med 2019;34:210219.

19. Brentano F, Schorr O, Gay RE, Gay S, Kyburz D. RNA released from necrotic synovial fluid cells activates rheumatoid arthritis synovial fibroblasts via Toll-like receptor 3. Arthritis Rheum 2005;52:2656-2665.

20. Kim SJ, Chen Z, Essani AB, et al. Identification of a novel toll-like receptor 7 endogenous ligand in rheumatoid arthritis synovial fluid that can provoke arthritic joint inflammation. Arthritis Rheumatol 2016;68:1099-1110.

21. Boyle WJ, Simonet WS, Lacey DL. Osteoclast differentiation and activation. Nature 2003;423:337-342.

22. Qureshi AA, Khan DA, Mushtaq S, Ye SQ, Xiong M, Qureshi N. $\delta$-Tocotrienol feeding modulates gene expression of EIF2, mTOR, protein ubiquitination through multiple-signaling pathways in chronic hepatitis $\mathrm{C}$ patients. Lipids Health Dis 2018;17:167.

23. Chang PN, Yap WN, Lee DT, Ling MT, Wong YC, Yap YL. Evidence of gamma-tocotrienol as an apoptosis-inducing, invasion-suppressing, and chemotherapy drug-sensitizing agent in human melanoma cells. Nutr Cancer 2009;61:357-366.

24. Feldmann M, Brennan FM, Foxwell BM, Maini RN. The role of TNF alpha and IL-1 in rheumatoid arthritis. Curr Dir Autoimmun 2001;3:188-199.

25. McInnes IB, Schett G. Cytokines in the pathogenesis of rheumatoid arthritis. Nat Rev Immunol 2007;7:429-442.

26. Guo YJ, Chen J, Xiong XG, Wu D, Zhu H, Liang QH. Effect of Bizhongxiao decoction and its dismantled formulae on IL-1 and TNF levels in collagen-induced arthritis in rat synovial joints. Theor Biol Med Model 2012;9:47.

27. Lee WS, Lee EG, Sung MS, Choi YJ, Yoo WH. Atorvastatin inhibits osteoclast differentiation by suppressing NF-кB and MAPK signaling during IL-1 $\beta$-induced osteoclastogenesis. Korean J Intern Med 2018;33:397-406.

28. Wu SJ, Liu PL, Ng LT. Tocotrienol-rich fraction of palm oil exhibits anti-inflammatory property by suppressing the expression of inflammatory mediators in human monocytic cells. Mol Nutr Food Res 2008;52:921-929. 